\title{
The apelin-13 influences the activity of pancreatic enzymes in young rats
}

\author{
H. Antushevich ${ }^{1,5}$, M. Kapica², A. Kuwahara ${ }^{3}$, I. Kato ${ }^{3}$, A. Krawczyńska', A.P. Herman', \\ B. Pawlina ${ }^{1}$ and R. Zabielski ${ }^{4}$ \\ ${ }^{1}$ The Kielanowski Institute of Animal Physiology and Nutrition Polish Academy of Sciences \\ Instytucka 3, 05-110 Jabłonna, Poland \\ ${ }^{2}$ University of Life Sciences, Faculty of Veterinary Medicine, Department of Animal Physiology \\ Akademicka 12, 20-950 Lublin, Poland \\ ${ }^{3}$ University of Shizuoka, Laboratory of Physiology, 422-8526 Shizuoka, Japan \\ ${ }^{4}$ Warsaw University of Life Sciences - SGGW, Faculty of Veterinary Medicine, Department of Physiological Sciences \\ Nowoursynowska 166, 02-766 Warsaw, Poland
}

KEY WORDS: apelin, CCK, lipase, pancreas, stomach, young rats

Received: 13 January 2016

Revised: $\quad 21$ April 2016

Accepted: 23 May 2016

${ }^{5}$ Corresponding author:

e-mail: antuszewicz@op.pl

\begin{abstract}
Apelin and its APJ receptor are present inter alia in colostrum and in the young animal gastrointestinal tract. This peptide exerts numerous effects participating in the appetite and drinking behaviour, gastric acid and insulin secretion. The aim of the study was to investigate the effect of apelin-13 on the activity of pancreatic and gastric enzymes in young animals. The two experiments were carried out on weaning Wistar rats (50 $\pm 10 \mathrm{~g})$ which received the apelin-13 or physiological saline (in the corresponding control groups) by intragastric or intraperitoneal way twice a day for 10 days (100 nmol $\cdot \mathrm{kg}^{-1}$ body weight). At the end of each experiment rats were sacrificed and blood samples were collected for apelin and cholecystokinin (CCK) radioimmunoassay. The fragments of the pancreas and stomach were weighted and frozen for the further digestive enzymes activity analysis. The intragastric and intraperitoneal administration of apelin-13 increased plasma CCK level in young rats. The intraperitoneal injection of apelin-13 stimulated pancreatic trypsin, a-amylase and lipase activity, but had no effect on the activity of gastric enzymes. On the other hand, the intragastric administration of apelin-13 stimulated only the activity of pancreatic lipase and had no effect on the activity of gastric pepsin and rennet. So, circulating apelin exerts the most pronounced effect on pancreatic enzymes activity in young rats, but neither circulating nor luminal apelin influences gastric enzymes activity. Regardless the route of administration, apelin stimulates lipase activity which points out its considerable role in the regulation of the fat digestion.
\end{abstract}

\section{Introduction}

The physiological processes in the young organisms run differently in comparison with the adult individuals. These differences can be observed in the nervous, endocrine, cardiovascular or respiratory systems (Godlewski et al., 2007). The proper development of all systems depends on the state of health of the gastrointestinal tract. A lot of disorders associated with the gastrointestinal tract functions 
lead to abnormal development of the whole organism. Sometimes the disorders acquired in childhood cause irreversible effects in adulthood. Therefore, the development of the gastrointestinal tract, especially of the functional digestive organs (e.g., pancreas, stomach and intestine), is an important aspect for the whole organism growth (Chandra and Liddle, 2009). Apelin and its apelin receptor APJ are widely expressed in the body (Tatemoto et al., 1998; Hosoya et al., 2000; Medhurst et al., 2003; Kapica et al., 2012). In the young rats APJ receptor is present in the epithelium, intestinal goblet cells and in smooth muscle cells (Wang et al., 2009). In the gastrointestinal tract of young rats the mRNA expression of apelin and APJ receptor is the highest at birth and decreases postnatally (Wang et al., 2009). In the postnatal rat stomach, the density of gastric apelin-containing cells increases progressively during weaning and then decreases in the adulthood (Wang et al., 2009).

Firstly, apelin was isolated from the bovine stomach extracts (Tatemoto et al., 1998). Now it is known that apelin is present in the body in many bioactive isoforms consisting of 12,13,17 or 36 amino acids and in the form of the pyroglutamated apelin-13 (Pyr(1)-apelin-13) (Medhurst et al., 2003). Apelin-36, apelin-17 and apelin-13 are the predominant molecular forms of endogenous hypothalamic and plasma apelin in rats (De Mota et al., 2000; Kawamata et al., 2001; Castan-Laurell et al., 2011). On the other hand, $\operatorname{Pyr}(1)$-apelin-13 was found to be a predominant isoform in human cardiac tissue (Maguire et al., 2009). All apelin isoforms bind to the apelin receptor APJ (Kawamata et al., 2001) that is a 7-transmembrane domain $\mathrm{G}_{\mathrm{i}}$-coupled receptor containing 380 amino acids (O'Dowd et al., 1993).

In adult organism apelin regulates food intake (Taheri et al., 2002) and stimulates cholecystokinin (CCK) secretion (Wang et al., 2009; Castan-Laurell et al., 2011). It also inhibits the insulin response to intravenous glucose in mice fed high-fat diet (CastanLaurell et al., 2011). The experiment conducted on IEC-6 cell line showed that apelin suppresses apoptosis, mitosis and DNA repair enzyme 8-oxoguanine (OGG 1/2) expression in the intestinal crypts (Antushevich et al., 2014). In adult rats apelin exhibited positive effect on the gastrointestinal tract rebuilding and regeneration (Antushevich et al., 2013), in contrast to young rats (Antushevich et al., 2015). Apelin occurs in colostrum, milk (Mesmin et al., 2011) and in the lumen of the gastrointestinal tract in young animals (Wang et al., 2009), which suggests presumable role of this peptide in gastrointestinal tract functioning in young organism.
Therefore, the aim of the present study was to investigate the effect of exogenous apelin administrated by different ways on gastric and pancreatic enzymes activity in young rats.

\section{Material and methods}

\section{Animals}

Two experiments were carried out on weaning male Wistar rats. In the first experiment rats received apelin intragastricly and in the second one intraperitonealy. Both experiments were approved by the II Local Ethics Committee for the Experiments on Animals in Lublin (Poland), agreement No. 49/2008. The rats were maintained in individual metabolic cages in a temperature controlled room $21 \pm 01{ }^{\circ} \mathrm{C}$ with a $12 \mathrm{~h} \mathrm{light/dark} \mathrm{cycle} \mathrm{(light} \mathrm{phase}$ 7:00 - 19:00) with free access to standard feed (Labofeed H Standard, Feed Company 'Morawski', Kcynia, Poland) and water. In each experiment the rats $(50 \pm 10 \mathrm{~g}$ of body weight (BW)) were divided into control and experimental group of 6 rats each. Apelin-13 (Hokuriku University, Japan) was given intragastricly (i.g.), using probe Agnthos (Lidingö, Sweden) or intraperitonealy (i.p.), using syringe for insulin injection, in a dose $100 \mathrm{nmol} \cdot \mathrm{kg}^{-1} \mathrm{BW}$ twice a day (9:00 and 21:00) for 10 days. Control groups received physiological saline in the corresponding way. Apelin dose was selected on the basis of our previous studies (Kapica et al., 2012; Antushevich et al., 2014). At the end of experiment, about $12 \mathrm{~h}$ after the last apelin-13 administration, the rats were weighed to monitor animal growth and euthanized with carbon dioxide. Blood samples were collected immediately afterwards directly from a heart in a volume of $1 \mathrm{ml}$ for apelin and CCK analysis. The blood samples were preserved using 10\% EDTA (Sigma-Aldrich, St. Louise, MO, USA), centrifuged for $10 \mathrm{~min}$ at $4500 \mathrm{rpm}$ at $4{ }^{\circ} \mathrm{C}$ and obtained plasma was stored at $-20{ }^{\circ} \mathrm{C}$. After blood collections the rats were subjected to autopsy and there were no abnormalities in the examined organs observed. The liver, stomach and pancreas were isolated and weighted. Liver was taken for weighting as the organ which plays a key role in the organism detoxification and production of biologically active compounds necessary for digestion, while pancreas and stomach were collected to study their enzymatic activity as in these organs digestive enzymes are produced. Next, the samples of pancreas and stomach tissues were frozen in liquid nitrogen and stored at $-80{ }^{\circ} \mathrm{C}$ until further assays. 


\section{Apelin and CCK concentration in plasma}

Total plasma concentration of apelin and CCK was measured by radioimmunoassay (RIA) using a commercially available kits: Apelin-36 (Rat, Mouse) and CCK (Phoenix Pharmaceutical Inc., Karlsruhe, Germany). The used kit for apelin examination is designed to measure overall concentration of apelin in blood because it detects all molecular isoforms of apelin. In the CCK RIA kit a nonsulphated CCK (26-33) octapeptide fragment was used as a standard. Both tests were conducted according to producer's recommendations.

\section{Pancreatic enzymes activity}

To evaluate the enzymatic activity, pancreatic samples were homogenized in a lysis buffer and in the obtained homogenates total protein content, and the activities of trypsin, amylase and lipase were analysed. Total protein content was measured using the Lowry method (Lowry et al., 1951) modified to be performed in 96-microwell plates. Trypsin activity was determined by a Trypsin Activity Assay Kit (Bio Vision, Milpitas, CA, USA); $\alpha$-amylase activity was measured by a QuantiChrom ${ }^{\mathrm{TM}} \alpha$-Amylase Assay Kit DAMY-100 (Bioassays Systems, Hayward, CA, USA) and lipase activity was determined using a Lipase-PS kit QuantiChrom ${ }^{\mathrm{TM}}$ Lipase Assay Kit (Bioassays Systems, Hayward, CA, USA). Tests were conducted according to the producers' recommendations.

\section{Gastric enzymes activity}

For gastric enzymes activity analysis a whole stomach was homogenized in a lysis buffer and in the obtained homogenates total protein, and the activities of pepsin and rennet were analysed. Total protein content was measured by the Lowry method as described above. Pepsin activity was determined according to the Walker method (Walker and Taylor, 1979). Rennet activity was measured according to Erlanger method using BAPNA (N- $\alpha$-benzoyl-DL-arginine- $p$-nitroanilide; SigmaAldrich, St. Louise, MO, USA) as a substrate (Erlanger et al., 1961).

\section{Statistical analysis}

Results are expressed as mean \pm standard error of mean (SEM). The Student's t-test was used to indicate the statistical differences between control and experimental group for each experiment separately (Statistica v.10.0, 2011; StatSoft Inc., Tulsa, OK, USA). In all statistical analyses $P<0.05$ was taken as the level of significance.

\section{Results}

\section{Influence of apelin-13 on the liver, stomach, pancreas and body weight}

The intragastric administration of apelin-13 increased $(P<0.001)$ stomach weight in comparison with the control group. No effect of intragastric and intraperitoneal apelin-13 administration on the liver, pancreas and final body weight was found (Table 1).

\section{Effect of apelin-13 on the plasma concentration of apelin and CCK}

In the young rats i.g. and i.p. administration of apelin-13 increased $(P<0.001)$ CCK level in blood. On the other hand, neither i.g. nor i.p. treatment with apelin-13 modified the overall plasma level of apelin (Table 2).

Table 1. Body and gastrointestinal organs weights of young rats after intragastric and intraperitoneal administration of apelin-13 (100 nmol $\cdot \mathrm{kg}^{-1}$ body weight / twice a day for 10 days)

\begin{tabular}{|c|c|c|c|c|}
\hline \multirow{2}{*}{ Experiment } & \multicolumn{4}{|c|}{ Weight, $\mathrm{g}$} \\
\hline & body & liver & stomach & pancreas \\
\hline \multicolumn{5}{|c|}{1 - intragastric administration } \\
\hline control & $84.7 \pm 13.1$ & $4.3 \pm 0.86$ & $0.744 \pm 0.07$ & $0.558 \pm 0.09$ \\
\hline apelin & $96.3 \pm 9.3$ & $5.1 \pm 0.65$ & $0.907 \pm 0.09^{* * *}$ & $0.520 \pm 0.12$ \\
\hline \multicolumn{5}{|c|}{2 - intraperitoneal injection } \\
\hline control & $84.7 \pm 12.1$ & $4.7 \pm 0.63$ & $0.836 \pm 0.11$ & $0.565 \pm 0.12$ \\
\hline pelin & $85.5 \pm 7.4$ & $4.6 \pm 0.66$ & $0.900 \pm 0.05$ & $0.550 \pm 0.14$ \\
\hline
\end{tabular}

values are given as means \pm SEM $(n=6) ;{ }^{* * *}$ - value significantly different at $P<0.001$

Table 2. Plasma apelin and cholecystokinin (CCK) concentration in young rats after intragastric and intraperitoneal administration of apelin-13 (100 nmol $\cdot \mathrm{kg}^{-1}$ body weight / twice a day for 10 days)

\begin{tabular}{lll}
\hline \multirow{2}{*}{ Experiment } & \multicolumn{2}{c}{ Plasma concentration, pmol $\cdot \mathrm{r}^{-1}$} \\
\cline { 2 - 3 } & apelin & $\mathrm{CCK}$ \\
\hline 1 - intragastric administration & \\
control & $5849 \pm 274$ & $51.09 \pm 1.9$ \\
apelin & $5933 \pm 199$ & $62.42 \pm 5.4^{* * *}$ \\
2 - intraperitoneal injection & & \\
control & $6218 \pm 227$ & $60.22 \pm 8.6$ \\
apelin & $5936 \pm 122$ & $78.64 \pm 7.6^{* * * *}$ \\
\hline
\end{tabular}

values are given as means \pm SEM $(n=6) ;{ }^{* * *}-$ value significantly different at $P<0.001$

\section{Influence of apelin-13 on the protein content and activity of pancreatic enzymes}

The intragastric administration of apelin-13 increased $(P<0.001)$ lipase activity in rat pancreatic homogenates by $47 \%$ (Figure 1D). No effect of i.g. administration of apelin-13 on the total protein content and the activity of trypsin and amylase was observed in comparison with the control group (Figure 1 - A,B,C). 

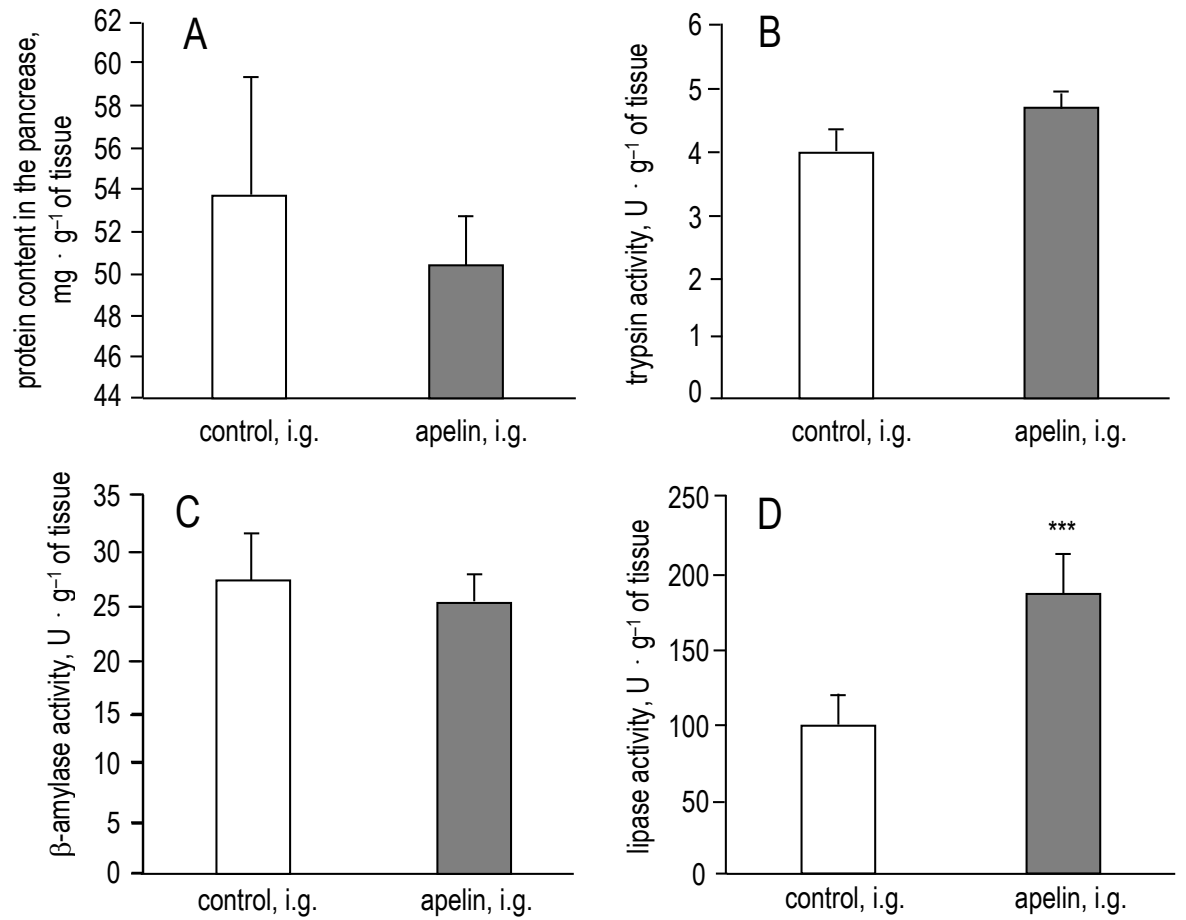

Figure 1. Protein content (mg $\cdot \mathrm{g}^{-1}$ of tissue) (A) and pancreatic enzymes: trypsin (B), amylase (C), lipase (D) activity (U $\cdot \mathrm{g}^{-1}$ of tissue) in rats after intragastric (i.g.) administration of apelin-13 (experiment 1). Values are given as means \pm SEM $(n=6)$; ${ }^{* *}$ - value significantly different at $P<0.001$
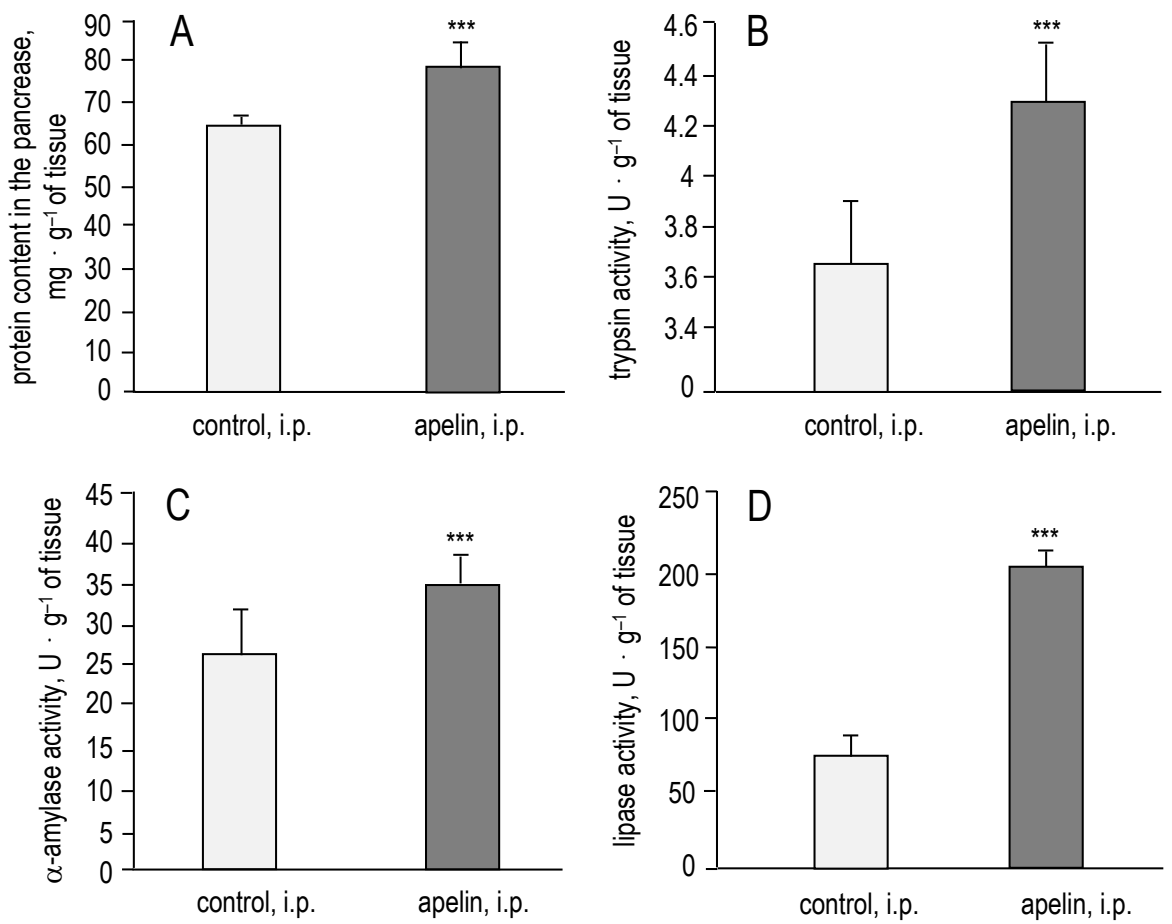

Figure 2. Protein content ( $\mathrm{g} \cdot \mathrm{kg}^{-1}$ of tissue) (A) and pancreatic enzymes: trypsin (B), amylase $(C)$, lipase (D) activity $\left(\mathrm{U} \cdot \mathrm{g}^{-1}\right.$ of tissue) in rats after intraperitoneal (i.p.) administration of apelin-13 (experiment 2). Values are given as means \pm SEM $(n=6)$; ${ }^{* *}-$ value significantly different at $P<0.001$

On the other hand, i.p. administration of apelin-13 increased $(P<0.001)$ all examined parameters in pancreas tissue. In the experimental group the protein content and activities of trypsin and $\alpha$-amylase were higher by 22,13 and $33 \%$, respectively, in comparison with the control group (Figure $2-A, B, C$ ). But the most pronounced effect was observed on pancreatic lipase activity $159 \%$ increase was by in comparison with the control group (Figure 2D). 
The effect of apelin-13 administration on the activity of gastric enzymes

In young rats, both i.g. and i.p. administration of apelin-13 exerted no effect on total protein content and the activity of pepsin and rennet in the young rats stomach tissue as compared with the corresponding control groups. After i.g. administration of apelin-13 the values were as follows: protein content $67.6 \pm 5.5 \mathrm{mg} \cdot \mathrm{g}^{-1}$ of tissue in the control group and $69.9 \pm 5.7 \mathrm{mg} \cdot \mathrm{g}^{-1}$ of tissue in the experimental group; the activity of pepsin $3.1 \pm 0.28 \mathrm{U} \cdot \mathrm{g}^{-1}$ of tissue in the control group and $3.5 \pm 0.5 \mathrm{U} \cdot \mathrm{g}^{-1}$ of tissue in the experimental group; the activity of rennet $40.6 \pm 7.7 \mathrm{U} \cdot \mathrm{g}^{-1}$ of tissue in the control group and $43.0 \pm 7.2 \mathrm{U} \cdot \mathrm{g}^{-1}$ of tissue in the experimental group. Similarly, in the experiment which studied the effect of i.p. administration of apelin-13 the values were as follows: protein content $59.4 \pm 8.9 \mathrm{mg} \cdot \mathrm{g}^{-1}$ of tissue in the control group and $62.1 \pm 6.9 \mathrm{mg} \cdot \mathrm{g}^{-1}$ of tissue in the experimental group; the activity of pepsin $3.34 \pm 0.33 \mathrm{U} \cdot \mathrm{g}^{-1}$ of tissue in the control group and $3.02 \pm 0.29 \mathrm{U} \cdot \mathrm{g}^{-1}$ of tissue in the experimental group; the activity of rennet $41.3 \pm 4.0 \mathrm{U} \cdot \mathrm{g}^{-1}$ of tissue in the control group and $38.6 \pm 5.7 \mathrm{U} \cdot \mathrm{g}^{-1}$ of tissue in the experimental group.

\section{Discussion}

The obtained results show that neither intraperitoneal (i.p.) nor intragastric (i.g.) administration of apelin influenced the body weight and studied gastrointestinal organs weights except stomach after i.g. administration. We supposed that the increased stomach weight may be connected with apelin indirect action on stomach smooth muscle layer proliferation. Literature data show that apelin-13 can stimulate vascular smooth muscle cell proliferation by promoting the G1-S phase transition, and that this effect is mediated in part by an apelin-pERK1/2cyclin D1 signal cascade (Li et al., 2008).

The present study also demonstrates that in young rats i.g. and i.p. administration of apelin-13 increases the level of plasma cholecystokinin (CCK), but has no effect on the overall plasma apelin level. It is known that CCK plays an important role in the mediation of gastrointestinal functions. It exerts effect on the gallbladder contraction which influences bile salts secretion and also increases blood flow and pancreatic enzymes secretion. CCK has also influence on duodenal secretion, satiety, insulin secretion and glucose homoeostasis (Rehfeld, 1989). Increased CCK concentration in blood in our experiment may be connected with stimulating actions of apelin on neurohormonal mediators and neurotrans- mitters, which activates CCK secretion. It was also previously found that regulatory peptides including apelin (Wang et al., 2009), leptin (Sobhani et al., 2002), ghrelin (Kapica et al., 2006; Nawrot-Porąbka et al., 2007) and obestatin (Kapica et al., 2007) when secreted into the gastric lumen, may reach the intestinal lumen and stimulate CCK secretion (Zabielski et al., 1998). In in vitro studies it was observed that apelin stimulated CCK secretion from a murine enteroendocrine cell line by mitogen-activated protein kinase (MAPK) pathway. Apelin also caused CCK release from dispersed intestinal endocrine cells and proximal small intestine cells ( $\mathrm{Lv}$ et al., 2013). No effect of experimental treatment on the plasma level of apelin may be due to the short half-life (about $8 \mathrm{~min}$ ) of plasma apelin (Aydin et al., 2014). In the presented experiment the blood was collected $12 \mathrm{~h}$ after the last apelin administration. We did not collect blood samples intravitally, because the procedure of blood taking is very stressful for young rat.

We also noticed that neither i.p. nor i.g. administration of apelin had an effect on the gastric pepsin and rennet activity. Our previous study showed that i.g. and i.p. administration of apelin-13 in the stomach increased the number of cells expressing the enzyme 8-oxoguanine glycosylase (OGG 1/2) indicating DNA damage in the cells (Antushevich et al., 2015). Such data allow us to suppose that the lack of apelin effect on gastric enzymes secretion may be connected with increased damage of cells responsible for secretion of pepsin and rennet in stomach.

Our present data also show that the i.p. administration of apelin-13 stimulates total protein content and the activity of all studied pancreatic enzymes (trypsin, $\alpha$-amylase and lipase), whereas the i.g. administration of apelin-13 rats stimulates only the activity of pancreatic lipase. The mechanism of apelin action on the gastrointestinal enzyme secretion is still unknown. Pancreatic juice secretion is regulated by endocrine, paracrine and neuronal interactions involving several regulatory peptides and neurotransmitter secreted in the gut (Holst et al., 1984; Sheikh et al., 1988; Dunning et al., 1990; Novak, 2003; Lee et al., 2012). However, the secretory function of pancreas in young individuals is insufficient. The lack of i.g. apelin-13 administration effect on the pancreatic enzymes activity (except lipase) obtained in the present study might be related to the lack of fully developed neurohormonal mechanisms or weak enteric nervous system development in young organism. Kapica et al. (2012), who studied the effect of apelin administration on the pancreato-biliary juice secretion, demonstrated that intravenous injection of apelin reduced pancreato-biliary juice volume and 
pancreatic output while the intraduodenal boluses of apelin led to the dose-dependent stimulation of pancreatic juice volume and protein output. In turn in in vitro experiment conducted on dispersed acinar cells obtained from rat pancreas, the highest dose of apelin-13 $\left(10^{-6} \mathrm{M}\right)$ stimulated basal and CCK-8-stimulated amylase release, whereas its lower doses $\left(10^{-7}-10^{-8} \mathrm{M}\right)$ were ineffective (Kapica et al., 2012). The impact of other regulatory peptides synthetized in stomach on pancreatic secretion is also depended on the way of their administration. Leptin or ghrelin given intravenously reduced pancreatic secretion, but given intraduodenally caused the opposite effect (Kapica et al., 2008). Stimulatory action of i.p. administrated apelin may be connected with circulating apelin influence on other biologically active compounds in blood which activate signalling pathways involved in the pancreatic enzymes secretion. In this study the observed effect of apelin on CCK secretion can explain increased lipase activity in young rats. The fact that apelin treatment stimulated the lipase activity in young rats suggests that apelin may influence the process of lipids digestion. Lipase is an enzyme that hydrolyses lipids forming fatty acids and glycerol. It is known that milk lipids provides about $40-60 \%$ energy demand for young animals therefore the increased pancreatic lipase activity in young rats after apelin administration may be connected with physiological need to digest milk lipids to provide the energy necessary for growth. Moreover, it was shown that in human apelin content in milk is higher than in colostrum, which is accompanied by an increased milk fat content (Aydin, 2010). Also, in our earlier studies in the same experimental arrangement conducted only on adult rats, the stimulatory effect of apelin-13 on the activity of lipase was found (Antushevich et al, 2016). In the above mentioned experiment, rats with removed main part of stomach producing apelin were also examined. It was observed that fundectomized rats exhibited the decreased apelin and CCK levels and pancreatic enzymes activity, which may confirm the of apelin in food digestibility. Such observation could suggest that apelin supplementation can be useful in patients with gastrointestinal diseases, however further studies in this field are needed. Higuchi et al. (2007) also stated that apelin participates in regulation of body adiposity. They showed in the experiment conducted on mice that daily i.p. apelin injections for 2 weeks decreased the triglycerides content in adipose tissue and the weight of different fat depots in both control and high-fat fed mice.

\section{Conclusions}

Circulating apelin exerts the most pronounced effect on pancreatic enzymes activity in young rats, but neither circulating nor luminal apelin has an effect on gastric enzymes activity. Regardless the route of administration apelin increases plasma CCK and pancreatic lipase activity suggesting a considerable role of apelin in the regulation of fat digestion. However, further studies are necessary to explain the mechanism of apelin action on lipid metabolism in young animals.

\section{Awcknowlegments}

This study was supported by the State Committee for Scientific Research (Poland), Grant No. N311 082737.

\section{References}

Antushevich H., Bierła J., Pawlina B., Kapica M., Krawczyńska A., Herman A.P., Kato I., Kuwahara A., Zabielski R., 2015. Apelin's effects on young rat gastrointestinal tract maturation. Peptides 65, 1-5

Antushevich H., Kapica M., Krawczynska A., Herman A.P., Kato I., Kuwahara A., Zabielski R., 2016. The role of apelin in the modulation of gastric and pancreatic enzymes activity in adult rats. J. Physiol. Pharmacol. 67 (in press)

Antushevich H., Krawczyńska A., Kapica M., Herman A.P., Zabielski R., 2014. Effect of apelin on mitosis, apoptosis and DNA repair enzyme OGG 1/2 expression in intestinal cell lines IEC-6 and Caco-2. Folia Histochem. Cytobiol. 52, 51-59

Antushevich H., Pawlina B., Kapica M., Krawczynska A., Herman A.P., Kuwahara A., Kato I., Zabielski R., 2013. Influence of fundectomy and intraperitoneal or intragastric administration of apelin on apoptosis, mitosis, and DNA repair enzyme OGG1,2 expression in adult rats gastrointestinal tract and pancreas. J. Physiol. Pharmacol. 64, 423-428

Aydin S., 2010. The presence of the peptides apelin, ghrelin and nesfatin-1 in the human breast milk, and the lowering of their levels in patients with gestational diabetes mellitus. Peptides 31, 2236-2240

Aydin S., Eren M.N., Sahin I., Aydin S., 2014. The role of apelins in the physiology of the heart. Protein Pept. Lett. 21, 2-9

Castan-Laurell I., Dray C., Attané C., Duparc T., Knauf C., Valet P., 2011. Apelin, diabetes, and obesity. Endocrine 40, 1-9

Chandra R., Liddle R.A., 2009. Neural and hormonal regulation of pancreatic secretion. Curr. Opin. Gastroenterol. 25, 441-446

De Mota N., Lenkei Z., Llorens-Cortés C., 2000. Cloning, pharmacological characterization and brain distribution of the rat apelin receptor. Neuroendocrinology 72, 400-407

Dunning B.E., Havel P.J., Veith R.C., Taborsky G.J., 1990. Pancreatic and extrapancreatic galanin release during sympathetic neural activation. Am. J. Physiol. - Endocrinol. Metab. 258, e436-e444

Erlanger B.F., Kokowsky N., Cohen W., 1961. The preparation and properties of two new chromogenic substrates of trypsin. Arch. Biochem. Biophys. 95, 271-278 
Godlewski M.M., Hallay N., Bierła J.B., Zabielski R., 2007. Molecular mechanism of programmed cell health in gut epithelium of neonatal piglets. J. Physiol. Pharmacol. 58, 97-113

Higuchi K., Masaki T., Gotoh K., Chiba S., Katsuragi I., Tanaka K., Kakuma T., Yoshimatsu H., 2007. Apelin, an APJ receptor ligand, regulates body adiposity and favors the messenger ribonucleic acid expression of uncoupling proteins in mice. Endocrinology 148, 2690-2697

Holst J.J., Fahrenkrug J., Knuhtsen S., Jensen S.L., Poulsen S.S., Nielsen O.V., 1984. Vasoactive intestinal polypeptide (VIP) in the pig pancreas: role of VIPergic nerves in control of fluid and bicarbonate secretion. Regul. Pept. 8, 245-259

Hosoya M., Kawamata Y., Fukusumi S. et al., 2000. Molecular and functional characteristics of APJ. Tissue distribution of mRNA and interaction with the endogenous ligand apelin. J. Biol. Chem. 275, 21061-21067

Kapica M., Jankowska A., Antushevich H., Pietrzak P., Bierla J.B., Dembinski A., Zabielski R., 2012. The effect of exogenous apelin on the secretion of pancreatic juice in anaesthetized rats. J. Physiol. Pharmacol. 63, 53-60

Kapica M., Laubitz D., Puzio I., Jankowska A., Zabielski R., 2006. The ghrelin pentapeptide inhibits the secretion of pancreatic juice in rats. J. Physiol. Pharmacol. 54, 691-700

Kapica M., Puzio I., Kato I., Kuwahara A., Zabielski R., 2008. Role of feed-regulating peptides on pancreatic exocrine secretion. J. Physiol. Pharmacol. 59, 145-159

Kapica M., Zabielska M., Puzio I., Jankowska A., Kato I., Kuwahara A., Zabielski R., 2007. Obestatin stimulates the secretion of pancreatic juice enzymes through a vagal pathway in anaesthetized rats - preliminary results. J. Physiol. Pharmacol. $58,123-130$

Kawamata Y., Habata Y., Fukusumi S. et al., 2001. Molecular properties of apelin: tissue distribution and receptor binding. Biochim. Biophys. Acta. 1538, 162-171

Lee M.G., Ohana E., Park H.W., Yang D., Muallem S., 2012. Molecular mechanism of pancreatic and salivary glands fluid and $\mathrm{HCO}_{3}$ secretion. Phys. Rev. 92, 39-74

Li F., Li L., Qin X. et al., 2008. Apelin-induced vascular smooth muscle cell proliferation: the regulation of cyclin D1. Front. Biosci. 13, 3786-3792

Lowry O.H., Rosenbrough N.J., Farr A.L., Randall R.J., 1951. Protein measurement with the Folin phenol reagent. J. Biol. Chem. 193, 265-275

Lv S.-Y., Yang Y.-J., Chen Q., 2013. Regulation of feeding behavior, gastrointestinal function and fluid homeostasis by apelin. Peptides 44, 87-92

Maguire J.J., Kleinz M.J., Pitkin S.L., Davenport A.P., 2009. [Pyr $\left.{ }^{1}\right]$ apelin-13 identified as the predominant apelin isoform in the human heart: vasoactive mechanisms and inotropic action in disease. Hypertension 54, 598-604
Medhurst A.D., Jennings C.A., Robbins M.J. et al., 2003. Pharmacological and immunohistochemical characterization of the APJ receptor and its endogenous ligand apelin. J. Neurochem. 84, 1162-1172

Mesmin C., Fenaille F., Becher F., Tabet J.-C., Ezan E., 2011. Identification and characterization of apelin peptides in bovine colostrum and milk by liquid chromatography-mass spectrometry. J. Proteome Res. 10, 5222-5231

Nawrot-Porabka K., Jaworek J., Leja-Szpak A., Szklarczyk J., Macko M., Kot M., Mitis-Musioł M., Konturek S.J., Pawlik W.W., 2007. The effect of luminal ghrelin on pancreatic enzyme secretion in the rat. Regul. Pept. 143, 56-63

Novak I., 2003. ATP as a signaling molecule: the exocrine focus. News Phys. Sci. 18, 12-17

O'Dowd B.F., Heiber M., Chan A., Heng H.H.Q., Tsui L.-C., Kennedy J.L., Shi X., Petronis A., George S.R., Nguyen T., 1993. A human gene that shows identity with the gene encoding the angiotensin receptor is located on chromosome 11. Gene 136, 355-360

Rehfeld J.F., 1989. Cholecystokinin. In: G.M. Makhlouf, S.G. Schultz (Editors). Handbook of Physiology. Section 6: The Gastrointestinal System. Volume II: Neural and Endocrine Biology. Oxford University Press, New York, NY (USA), pp. 337-358

Sheikh S.P., Holst J.J., Skak-Nielsen T., Knigge U., Warberg J., Theodorsson-Norheim E., Hokfelt T., Lundberg J.M., Schwartz T.W., 1988. Release of NPY in pig pancreas: dual parasympathetic and sympathetic regulation. Am. J. Physiol. - Gastrointest. Liver Physiol. 255, G46-G54

Sobhani I., Buyse M., Goïot H., Weber N., Laigneau J.-P., Henin D., Soulé J.C., Bado A., 2002. Vagal stimulation rapidly increases leptin secretion in human stomach. Gastroenterology 122, 259-263

Taheri S., Murphy K., Cohen M., Sujkovic E., Kennedy A., Dhillo W., Dakin C., Sajedi A., Ghatei M., Bloom S., 2002. The effects of centrally administered apelin-13 on food intake, water intake and pituitary hormone release in rats. Biochem. Biophys. Res. Commun. 291, 1208-1212

Tatemoto K., Hosoya M., Habata Y. et al., 1998. Isolation and characterization of a novel endogenous peptide ligand for the human APJ receptor. Biochem. Biophys. Res. Commun. 251, 471-476

Walker V., Taylor W.H., 1979. Pepsin 5 in gastric juice: determination and relationship to the alkali-stable peptic activity. Gut 20 , 977-982

Wang G., Kundu R., Han S., Qi X., Englander E.W., Quertermous T., Greeley G.H. Jr., 2009. Ontogeny of apelin and its receptor in the rodent gastrointestinal tract. Regul. Pept. 27, 32-39

Zabielski R., Leśniewska V., Borlak J., Gregory P.C., Kiela P., Pierzynowski S.G., Barej W., 1998. Effects of intraduodenal administration of tarazepide on pancreatic secretion and duodenal EMG in neonatal calves. Regul. Pept. 78, 113-123 